\title{
La transformación curricular en Derecho: consideraciones desde la experiencia docente y de gestión académica
}

\section{Curriculum changes in law: considerations from teaching experience and academic management}

\author{
Dr. Rodrigo Palomo Vélez ${ }^{1}$ \\ Dra. Carolina Riveros Ferrada ${ }^{2}$
}

Resumen: Este trabajo expone una sistematización y evaluación del proceso de transformación curricular y la implementación del mismo en la carrera de Derecho de la Universidad de Talca (Chile), a partir de la experiencia de los autores en docencia y gestión académica.

La Universidad de Talca ha sido pionera en asumir como eje estratégico la transformación curricular de sus carreras de pregrado hacia un modelo basado en competencias, y ha ejecutado diversas acciones para implementar la redefinición de los perfiles de graduación, de los planes de estudio, y de estrategias metodológicas y sistemas de evaluación, cuando ha sido necesario.

El trabajo da cuenta de este proceso respecto de la carrera de Derecho de la Universidad de Talca, abordando los principales hitos ocurridos en la última década. Además, aporta la visión de académicos que han estado involucrados en el proceso tanto en la perspectiva docente como de gestión.

Palabras clave: transformación curricular, Universidad, Derecho, docencia y gestión académica.

Abstract: This paper presents the systematization, evaluation and implementation of the new law degree curriculum at University of Talca (Chile). The paper focuses on the authors' experience in teaching and academic management during this process.

University of Talca has pioneered in the transformation towards a competency-based model for its degrees. The university also has redefined graduate profiles, modified the curricula and used different methodological strategies and forms of evaluation when necessary.

This paper describes the law degree curriculum reform process at University of Talca pointing out the main challenges occurred during the last decade. It also shows a range of views of academics involved in the process, either teaching or carrying out managerial work.

Keywords: university curriculum reform, University, Law, teaching and academic management.

\footnotetext{
${ }^{1}$ Profesor de Derecho del Trabajo, Director del Magíster en Derecho del Trabajo y de la Seguridad Social, Facultad de Ciencias Jurídicas y Sociales, Universidad de Talca (Chile). Correo electrónico: rpalomo@utalca.cl

${ }^{2}$ Profesora de Derecho Civil, Directora de la Escuela de Derecho - Santiago, Facultad de Ciencias Jurídicas y Sociales, Universidad de Talca (Chile). Correo electrónico: criveros@ utalca.cl
} 


\section{Marco de referencia: Universidad de Talca}

La Universidad de Talca es una Corporación de Derecho Público ${ }^{3}$. La reforma del sistema universitario de 1981 puso término a las sedes regionales que tenían la Universidad de Chile y la Universidad Técnica del Estado, las que fueron fusionadas en universidades autónomas o en institutos profesionales ${ }^{4}$. En marzo de 1981, las sedes correspondientes a la ciudad de Talca fueron fusionadas dando origen a un instituto profesional y posteriormente, en octubre del mismo año, a la Universidad, definida como una institución de educación superior, independiente, autónoma y con personalidad jurídica propia.

Conforme al Plan Estratégico 2015, la Universidad de Talca tiene como misión "la formación de personas dentro de un marco valórico. Busca la excelencia en el cultivo de las ciencias, las artes, las letras y la innovación tecnológica y está comprometida con el progreso y bienestar regional y del país, en permanente diálogo e interacción con el entorno social, cultural y económico, tanto local como global". Por su parte, ha definido como visión "ser reconocida como una universidad innovadora, internacionalizada, de excelencia, referente en el sistema educacional superior, pertinente en su accionar, social y ambientalmente responsable". Son valores corporativos declarados por la Universidad la tolerancia, pensamiento crítico, solidaridad, conciencia ambiental, honestidad, convicción democrática, responsabilidad social, profesionalismo, responsabilidad y sensibilidad estética.

\section{La Facultad de Ciencias Jurídicas y Sociales y la carrera de Derecho}

La Facultad de Ciencias Jurídicas y Sociales y la carrera de Derecho nacen en 1992, constituyendo un hito relevante en la historia de la Universidad de Talca, toda vez que ésta fue su primera licenciatura en el área humanista y la primera Escuela de Derecho creada entre Santiago y Concepción.

Con más de dos décadas de trayectoria, la Facultad imparte actualmente la carrera de Derecho en Talca (desde 1992), con más de 800 estudiantes, y en Santiago (desde 2012), con más de 200 alumnos regulares. Además, imparte la carrera de Administración Pública (desde 2013).

Conforme al Plan de Contribución de la Facultad de Ciencias Jurídicas y Sociales al Plan Estratégico de la Universidad de Talca 2011-2015, la misión de la Unidad "es el cultivo de

\footnotetext{
${ }^{3}$ Creada el 26 de octubre de 1981 por medio del Decreto con Fuerza de Ley $\mathrm{N}^{\circ}$ 36, del Ministerio de Educación Pública. La Casa Central de la Institución está ubicada en Talca, capital de la Región del Maule. Posee un campus en el sector nororiente de esta ciudad, denominado Campus Talca (1981); otro en Curicó (1995), para las Carreras de Ingeniería; un campus en Santiago para postgrados, extensión e investigación (2004) y para las Carreras de Derecho, Ciencia Política y Administración Pública, Ingeniería Comercial, Auditoría e Ingeniería en Control de Gestión (2012); y un campus en Santa Cruz destinado a la formación de técnicos de nivel superior en el área vitivinícola (2007).

${ }^{4}$ Un interesante trabajo acerca de la realidad de la educación superior en torno al territorio en DONOSO, S., ARIAS, O., WEASON, M., FRITES, C. "La oferta de educación superior de pregrado en Chile desde la perspectiva territorial: inequidades y asimetrías en el mercado" en revista Calidad en la Educación, 2012, núm. 37. P. 99-127.
} 
las ciencias jurídicas, sociales, políticas y administrativas, lo que desarrolla a través de la dictación de programas de formación académica y profesional de pregrado y postgrado en las disciplinas respectivas; la generación, aplicación y difusión del conocimiento científico; la promoción del debate universitario multidisciplinario en materia de políticas públicas; y el desarrollo y promoción de una cultura jurídica y política humanista y democrática". La Facultad concreta esta misión a través de programas académicos de pregrado y postgrado, de investigación y de extensión, en la búsqueda de satisfacer las necesidades nacionales y del entorno regional, dentro del marco exigido por la excelencia académica.

\section{Antecedentes del proceso de rediseño curricular: antecedentes institucionales}

En el año 2003 se dio inicio, como una apuesta estratégica de la Universidad de Talca, a la ejecución del proyecto MECESUP Tal0101 denominado "Construcción e instalación de una visión renovada de la formación de pregrado. Rediseño y validación de los currícula de las carreras profesionales", que -entre otros aspectos- impulsó el rediseño de los planes de formación de todas las carreras de pregrado a un modelo de formación basado en competencias profesionales.

En este contexto, la Resolución Universitaria (en adelante, RU) 082, de 18 de enero de 2005, que promulga el Acuerdo $\mathrm{N}^{\mathrm{0}} 734$ de la Junta Directiva de la Universidad, que aprueba el Perfil Genérico y la Estructura Curricular del Grado de Licenciado y del Título Profesional, definió a nivel central las etapas de todo plan de formación (dos ciclos); las diversas áreas de formación y; la determinación y distribución de los créditos ECTS ${ }^{5}$.

En términos sencillos, el cambio en el modelo educativo impulsado desde 2003 en la Universidad de Talca ha supuesto un cambio en:

- $\quad$ Los perfiles profesionales.

- $\quad$ Los curriculum (itinerarios curriculares o planes de formación).

- Los programas y syllabus (objetivos de aprendizaje).

- $\quad$ La metodología de enseñanza-aprendizaje (en cuanto sea pertinente).

- $\quad$ El sistema de medición y evaluación (de estudiantes y docentes).

El rediseño curricular obedeció, por tanto, a una decisión institucional estratégica de reformulación de las carreras de pregrado al modelo de educación basada en competencia ${ }^{6}$. Tras la transformación curricular (2003-2005) y la instalación de los planes de formación rediseñados (2005-2012), actualmente la Universidad de Talca se encuentra desarrollando un proceso de armonización curricular (2013-2015), concentrado en la revisión de los

\footnotetext{
${ }^{5}$ En relación con una visión crítica de la gestión universitaria, véase FERNANDEZ, E; STOCK, M Los límites de la gestión. Consideraciones sociológicas sobre la gestión universitaria en Chile y Alemania. Revista Universum 2007, N²2 Vol.2: P.108-124.

${ }^{6}$ Una sistematización del proceso de rediseño curricular en la carrera de Derecho y de los primeros años de implementación puede revisarse en ACUÑA SAN MARTÍN, Marcela; PALOMO VÉLEZ, Rodrigo. Transformación curricular basada en competencias para la carrera de Derecho. Un cambio necesario. Editorial Universidad de Talca, 2009. 71 p.
} 
perfiles y planes de formación, y en la adecuación del modelo educativo a los resultados de la autoevaluación tras el egreso de las primeras cohortes rediseñadas ${ }^{7}$.

Con dicho propósito, los Comités Curriculares de las distintas carreras han venido realizando diversas tareas, entre las que cabe mencionar las siguientes: elaboración y análisis de ranking de tasas de aprobación de módulos, sistematización de la información sobre cohortes de licenciados rediseñados, elaboración de catastros de habilitación docente, talleres DACUM con empleadores, levantamiento y análisis de bitácoras de carga académica de estudiantes, análisis del estado del arte de las carreras en Chile, revisión analítica de los perfiles de licenciatura, análisis de la posibilidad de formación por ciclos o certificación de competencias intermedias, análisis de la posibilidad de incorporar compromisos de aprendizaje del idioma inglés, definición de trayectorias de aprendizaje y temporalidad a las mismas, entre otras.

El proceso está en plena concordancia con el Plan Estratégico Institucional (primer foco estratégico) y con el Plan de Contribución de la Facultad de Ciencias Jurídicas y Sociales a dicho Plan de la Universidad.

\section{El rediseño del perfil del Licenciado en Ciencias Jurídicas}

Como se ha señalado, la carrera de Derecho debió sumarse a la transformación curricular promovida desde el nivel central de la Universidad. Así pues, previa realización de estudios de necesidad y factibilidad, definió un nuevo perfil del licenciado y seguidamente un nuevo plan de formación.

En un modelo de educación basado en competencias, el perfil profesional constituye una descripción del conjunto de atributos de un licenciado en términos del ejercicio de una profesión, pudiendo ser descrito por las competencias asociadas a las prácticas de la misma.

A partir de un perfil genérico de pregrado procurado por la Universidad, nuestra Facultad definió el perfil del Licenciado en Ciencias Jurídicas en consideración, principalmente, de tres criterios:

1. Definición de dominios de competencias. Sin perjuicio de los diversos ámbitos y lugares de desempeño profesional de un abogado, se concluyó que su hacer característico está dado por la representación judicial y extrajudicial de intereses públicos y privados, así como por la asesoría jurídica de los mismos intereses.

2. Identificación de competencias y capacidades. Con la definición anterior, se desagregaron las competencias y capacidades asociadas, entendidas éstas como una compleja combinación de conocimientos, actitudes, habilidades y valores desplegados en el ambiente del desempeño de una tarea.

3. Áreas disciplinarias relevantes. En consideración de que las competencias no se estructuran en abstracto sino que respecto de las distintas disciplinas, la Facultad definió las áreas del Derecho que conforman el constructo o dimensión conceptual de la formación.

\footnotetext{
${ }^{7}$ Sobre los compromisos institucionales asumidos en virtud del Convenio de Desempeño en Armonización Curricular, véase http://convenios.utalca.cl/html/armonizacion/obj_general.html
} 
Estos criterios se tradujeron en un perfil conceptualizado del Licenciado en Ciencias Jurídicas de la Universidad de Talca, y su desagregación en un perfil desarrollado en sus competencias y capacidades. Ambos fueron validados por el cuerpo académico de la Facultad. Ellos explicitan el compromiso formativo que hemos asumido, y por tanto, deben ser los principios rectores o lineamientos de todas las decisiones que se tomen en esta materia.

El perfil conceptualizado del Licenciado es el siguiente:

"El Licenciado en Ciencias Jurídicas de la Universidad de Talca tiene un conocimiento sistemático de los principios generales e instituciones esenciales del Derecho Civil, Derecho Constitucional, Derecho Penal, Derecho Procesal, Derecho Comercial, Derecho Administrativo y Derecho del Trabajo. A ello se suma un conocimiento especial de los Derechos Fundamentales, del Derecho Internacional Público y Privado y del Derecho del Medio Ambiente.

Es capaz de analizar, argumentar, razonar y resolver problemas jurídicos, en consideración de la perspectiva ética y social, y posee las competencias profesionales indispensables para desempeñarse en la representación judicial y extrajudicial de intereses públicos y privados. En efecto, está en condiciones de establecer, con sus consecuencias, la situación jurídica actual y las alternativas de solución; redactar fundadamente los escritos necesarios para representar judicialmente los derechos del patrocinado o defendido; tramitar la acción o excepción respectiva; efectuar defensas orales ante los organismos que correspondan, y tramitar, gestionar, contratar y celebrar otros acuerdos con o ante organismos, personas y entidades. En la asesoría jurídica, en relación a los mismos intereses, está en condiciones de prestar servicios de asesoría jurídica consultiva y ejecutiva, va dando respuesta fundada a consultas o realizando las actividades necesarias a la satisfacción de los intereses involucrados.

Es capaz de comunicarse eficazmente e interactuar con otros, comprender el mundo y la sociedad en que vive y demostrar compromiso social y ciudadano".

Se trata de un producto colectivo, propio y distintivo de la personalidad de la Carrera. Consistente con la lógica del nuevo modelo curricular, se hace cargo, en sus tres párrafos, de la tridimensionalidad de las competencias profesionales, va dando cuenta de su compleja estructura y asumiéndolas como un propósito del proceso de enseñanza-aprendizaje que pretende un profesional integral en los conocimientos, los procedimientos y las actitudes. Además, deja ver la intencionalidad de nuestras decisiones, en cuanto al consorcio existente entre los desempeños profesionales específicos y los previos conocimientos disciplinarios que constituyen la plataforma de dichos desempeños, lo que es de suyo relevante pues salva los temores relativos a la posible traslación de la enseñanza científica a la enseñanza técnica del Derecho. Por último, el perfil del licenciado se apropia también del perfil genérico de la Universidad.

Dicho perfil fue recientemente revisado y modificado, a propósito del proceso de armonización curricular. En concreto, sin variar su lógica, optimizó su planteamiento, a eliminar reiteraciones inoficiosas. El nuevo perfil de licenciatura, en definitiva, fue aprobado por el Consejo de Facultad el 23 de junio de 2014, y es el siguiente: 
Competencia 1. Establecer la situación jurídica y las alternativas de solución frente a un conflicto jurídico, interpretando y aplicando el Derecho pertinente, a fin de quedar en condiciones de representar judicial o extrajudicialmente intereses públicos y/o privados.

Competencia 2. Prestar asesoría jurídica a personas u organismos, dando respuesta a sus consultas conforme al ordenamiento jurídico vigente, para prevenir o dar solución a un conflicto jurídico.

Competencia 3. Defender los intereses públicos y privados en el ámbito judicial, interpretando y aplicando el Derecho pertinente, a través de la litigación en tribunales, para solucionar conflictos jurídicos.

Competencia 4. Gestionar solicitudes ante organismos y/o celebrar acuerdos y contratos en el ámbito de la representación extrajudicial de intereses, para atender los requerimientos del asesorado.

\section{El rediseño del Plan de Formación}

Tras la validación y aprobación del perfil del licenciado vino el proceso operacionalización curricular, que consistió básicamente en definir y construir módulos que se comprometieran con el logro de las competencias y capacidades ya definidas en el perfil. Se procuró, en ese sentido, que cada uno de los módulos correspondiera a las unidades de trabajo/aprendizaje que, en su conjunto, configurarían el itinerario (o malla) curricular de la Carrera.

En concreto, este proceso tuvo dos grandes momentos interrelacionados: la definición de la organización curricular y la articulación de la estructura curricular.

El rediseño curricular en la Carrera se ha traducido en tres planes de formación, con enfoque en un modelo de educación basado en competencias: Plan 44/2006 y Plan 11/2011, con sus respectivas modificaciones, y recientemente, Plan 15/2015.

\section{a) Plan 44/2006 y sus modificaciones}

La RU 004, de 4 de enero de 2006, promulgó el Acuerdo № 803 de la Junta Directiva, que aprobó un nuevo plan de formación de la Carrera. Dicha norma universitaria recogió, entonces, la versión original del plan de formación rediseñado para Derecho.

El referido Plan, denominado 44, contemplaba dos ciclos:

- Primer ciclo, conducente al egreso: 10 semestres, 300 créditos ECTS, con módulos de formación fundamental, básica y disciplinaria (más 2 créditos ECTS de práctica deportiva).

- Segundo ciclo, conducente a la graduación: 1 semestre, 30 créditos ECTS, contenedor de la Actividad Final de Licenciatura (en adelante, AFL).

- $\quad$ El texto original del Plan 44 ha tenido las siguientes modificaciones:

- $\quad$ RU 015, de 4 de enero de 2008: modifica el nombre, creditaje, ubicación dentro del itinerario curricular y prerrequisitos de los módulos que indica; determina que los módulos de integración se desarrollen en modalidad intensiva, fuera del periodo lectivo; y modifica el carácter obligatorio por electivo de determinados módulos. 
- RU 286, de 24 de abril de 2009: modifica nombre, estructura y contenido de módulos que indica; flexibiliza prerrequisitos de diversos módulos; y reformula los talleres procedimentales.

- RU 1154, de 14 de diciembre de 2009: ajusta reformulación de talleres procedimentales; modifica nombre y creditaje de módulos que indica; y crea nuevos módulos.

- $\quad$ RU 117, de 1 de marzo de 2011: elimina módulo electivo de quinto año, por haber sido trasladado a cuarto.

- $\quad$ RU 915, de 1 de septiembre de 2011: modifica creditaje y prerrequisitos de módulos que indica; disminuye creditaje total de la Carrera (332 a 316); y suprime distinción entre primer y segundo ciclo de formación (elimina AFL y establece como requisito de graduación la aprobación de un examen de Licenciatura, con 30 créditos asignados).

- $\quad$ RU 471, de 18 de abril de 2012: modifica tipo de formación, distribución de trabajo presencial y no presencial, y prerrequisitos de módulos que indica.

El Plan 44 rige para los estudiantes que hayan ingresado a la carrera entre 2006 y 2010.

\section{b) Plan 11/2011 y sus modificaciones}

La RU 240, de 21 de marzo de 2011, promulgó el Acuerdo № 1171 de la Junta Directiva, que aprobó un nuevo plan de formación de la carrera de Derecho. Su aprobación obedeció fundamentalmente a la decisión institucional de revisar el Programa de Formación Fundamental (en adelante, PFF), administrado por la Vicerrectoría de Docencia de Pregrado, luego del egreso de la primera cohorte de aquel Programa. La reforma operada al PFF se tradujo, en lo medular, en una reducción del peso asignado a la formación fundamental y en un aumento consecuencial del peso de la formación básica ofrecida por las Escuelas. Por lo mismo, el perfil del licenciado no experimentó cambios ${ }^{8}$.

Este Plan, denominado Plan 11, también contemplaba originalmente la distinción entre dos ciclos de formación:

- Primer ciclo: 10 semestres, 276 créditos ECTS, con módulos de formación fundamental, básica y disciplinaria (más 2 créditos ECTS de práctica deportiva), conducía al egreso.

- Segundo ciclo: 1 semestre, 27 créditos ECTS, AFL, conducía a la licenciatura.

El texto original del Plan 11 ha tenido las siguientes modificaciones:

- $\quad$ RU 916, de 1 de septiembre de 2011: modifica creditaje y prerrequisitos de módulos que indica; y suprime distinción entre primer y segundo ciclo de formación (elimina AFL y establece como requisito de graduación la aprobación de un EL, con 27 créditos asignados $)^{9}$.

\footnotetext{
${ }^{8}$ En efecto, el Plan 11 no ha modificado el Perfil del Licenciado en Ciencias Jurídicas aprobado por la RU 004, de 4 de enero de 2006. Por el contrario, la RU 240, de 21 de marzo de 2011, lo reproduce íntegramente.

${ }^{9}$ Recuérdese que una modificación similar planteó la RU 915, de igual fecha, respecto del Plan 44.
} 
- RU 485, de 23 de abril de 2012: modifica distribución de trabajo presencial y no presencial, y prerrequisitos de módulos que indica.

El Plan 11 rige para los estudiantes que hayan ingresado a la carrera a partir de 2011, y para aquellos de ingresos anteriores que soliciten formalmente su adscripción a este Plan.

En síntesis, las principales innovaciones que ha incorporado el rediseño en los planes de formación que lo han concretado han sido las siguientes:

1. Coherencia y pertinencia de la formación básica en relación con el perfil comprometido.

2. Módulos con enfoque multidisciplinario.

3. Talleres procedimentales.

4. Módulos de desempeño integrado de competencias.

5. Módulos de integración de competencias antes del egreso.

6. Actividad final de licenciatura, sin perjuicio de sus diversas concepciones.

7. Consistencia de la duración de la carrera con la hipótesis de aprendizaje del estudiante (dos ciclos de formación).

No todas estas innovaciones subsisten en los planes de formación basados en competencias que rigen actualmente.

\section{c) Plan 15/2015}

La RU 2145, de 23 de diciembre de 2014, promulgó el Acuerdo No 1443 de la Junta Directiva, que aprueba el actual plan de estudios de la Carrera de Derecho de la Universidad de Talca, denominado Plan 15.

Este Plan es fruto del trabajo desarrollado a propósito de la ejecución de un convenio de desempeño sobre armonización curricular, que pretende optimizar los planes de estudio a partir de la revisión de la experiencia de su implementación en las primeras cohortes de ingreso. Al efecto, tras el diagnóstico y evaluación de la experiencia curricular, y la redefinición del perfil de graduación en los términos ya comentados, se elaboraron trayectorias de aprendizaje, entendidas éstas como el mapa de progreso en el tiempo del desarrollo lógico y armónico de cada competencia. Dichas trayectorias constituyen el sustrato básico del nuevo Plan.

El Plan 15 rige para los estudiantes que hayan ingresado a la carrera a partir de 2015, y para aquellos de ingresos anteriores que soliciten formalmente su adscripción a este Plan. 


\section{Implementación del rediseño curricular}

La Escuela de Derecho - Talca comenzó a implementar progresivamente el Plan de Formación 44 a partir del ingreso 2006. Por su parte, la implementación del Plan 11 comenzó el primer semestre de 2011. Por tanto, la Escuela de Derecho - Santiago ha impartido la Carrera con planes rediseñados desde su creación (2012). A partir de la cohorte de ingreso 2015, rige el Plan 15.

El segundo semestre de 2010 egresaron los primeros estudiantes formados bajo el nuevo modelo educativo, que reunió algunos de ellos los requisitos de licenciatura durante 2011. $\mathrm{Si}$ bien el número de licenciados del rediseño es todavía escaso, es posible plantear al menos un par de observaciones generales respecto del proceso de instalación del rediseño curricular en Derecho.

En primer término, es preciso relevar la importancia de las evaluaciones periódicas al proceso de implementación del rediseño en la Carrera, en las que intervienen principalmente el Director de Escuela, los Directores de Departamento y el Decano. En distinta medida, también se han involucrado en estas evaluaciones actores relevantes del proceso (docentes y estudiantes). El objetivo de las mismas ha sido la evaluación de estado de avance del rediseño (gestión administrativa y docente) y la promoción de las modificaciones que se han estimado pertinentes. Conviene mantener estas evaluaciones periódicas en promoción de una participación más activa de profesores y estudiantes, no sólo cuando se detectan situaciones especialmente conflictivas, sino en todos los casos; ellas pueden constituir un espacio fértil para la reapropiación del modelo formativo. Asimismo, cabe señalar que se han ido expandiendo sus propósitos a otros de mayor alcance, lo que ha permitido, por ejemplo, la evaluación colectiva de las estrategias metodológicas.

En segundo lugar, es necesario dar cuenta de la disparidad que ha presentado el acompañamiento docente en las distintas etapas del proceso de instalación del rediseño curricular, en lo referente a la inducción al modelo de educación basada en competencias, la elaboración colectiva e intencionada de syllabus y pautas de evaluación de desempeño, la construcción de instrumentos de evaluación de competencias, y la discusión de estrategias metodológicas. En efecto, el Comité Curricular de la Carrera continuó trabajando intensamente los primeros años de la implementación, denominándose en esta etapa "Comisión de apoyo a la instalación curricular". Luego, el acompañamiento docente se fue desintegrando hasta remitirse en la actualidad, casi exclusivamente, al apoyo -por cierto relevante- de las Direcciones de Escuela.

Por su parte, el apoyo institucional en estas materias también ha sido decreciente. Si bien subsiste la oferta del Diplomado en Educación Superior basada en Competencias, impartido por la Vicerrectoría de Docencia de Pregrado, éste plantea diversas restricciones que dificultan la incorporación de profesores de nuestra Facultad. Lo propio ocurre con el soporte técnico prestado por el Centro de Innovación y Calidad de la Docencia (en adelante, CICAD) y la desarticulación de interesantes experiencias, como la Red de Prácticas Docente. 


\section{Evaluación del rediseño curricular en la carrera de Derecho Planes de Formación e itinerarios curriculares}

El Plan de Formación 44, en su versión original, fue una apuesta innovadora seria, fruto del trabajo colectivo de los profesores de la Facultad, con el apoyo de expertos. Con todo, evidenció algunas falencias a poco andar del proceso de implementación, las que pudieron deberse a diversos factores, entre los cuales cabe mencionar los siguientes:

- $\quad$ El Plan de Formación rediseñado, en su versión inicial, y concretamente el itinerario curricular allí previsto, representó una hipótesis del recorrido de aprendizaje que debían desarrollar los estudiantes para alcanzar el perfil del licenciado. Como toda hipótesis, se ha sometido a comprobación durante el proceso de implementación del rediseño, es ésta la oportunidad natural para efectuar los ajustes necesarios, como ha venido ocurriendo.

- La necesidad de acuerdos mayoritarios que dieran legitimidad interna al Plan de Formación que se aprobaba direccionó determinadas decisiones que no se ajustaban plenamente al modelo de educación basado en competencias (ej. demandas de mantención del peso relativo de diversas disciplinas jurídicas).

- Nuestra inexperiencia en procesos de esta naturaleza y envergadura pudo permitir cierto voluntarismo al concebir módulos de difícil concreción práctica, sea por la ausencia de profesores (o equipos docentes) con la experticia necesaria para desarrollarlos, sea por la indefinición de las competencias que se comprometían en ellos (ej. "Enfoque multidisciplinario del negocio jurídico").

- Vinculado a lo anterior, la construcción de syllabus dejó en evidencia el -a vecesdifícil tránsito desde el proceso de modularización hasta la definición concreta del contenido de cada módulo, cuestión que supuso especial problema a la hora de definir la AFL.

Varias de las falencias o debilidades del Plan de Formación 44 fueron detectadas oportunamente en las sucesivas evaluaciones del proceso de implementación del rediseño, y solucionadas en su mayoría a través de modificaciones y/o cambios del Plan de Formación original $^{10}$.

Una cuestión que ha requerido varias revisiones ha sido la determinación de los prerrequisitos de los módulos. En efecto, la ubicación de los módulos en el itinerario curricular no es aleatoria. Por el contrario, se ha de tener en cuenta la hipótesis evolutiva

\footnotetext{
10 "De esta manera, el sistema universitario se desprende de su cuota de responsabilidad sobre los resultados educativos del estudiante y "privatiza" el fracaso (repitencia y deserción) como una consecuencia del mismo estudiante y no como un proceso en el cual a la universidad le corresponde la provisión de los recursos adecuados (docentes, infraestructura, equipamiento) para que el alumno alcance los aprendizajes esperados, algo que incluye también las políticas compensatorias". Con los diferentes y constantes análisis en la Universidad y especialmente, en las Escuelas de Derecho, se intenta que no sólo sea un problema personal de cada estudiante sino un problema asumido por las entidades universitarias correspondientes. DONOSO, S., SCHIEFELBEIN, E “Análisis de los modelos explicativos de retención de estudiantes en la universidad: una visión desde la desigualdad social” Estudios pedagógicos xxxiii, n 1: 7-27, 2007.
} 
del estudiante, en orden a permitirle que a través de la aprobación secuenciada y progresiva de los diferentes módulos logre evidenciar el perfil del Licenciado en Ciencias Jurídicas.

En esta materia se han detectado diversos problemas que se han intentado solucionar en las sucesivas modificaciones a los planes de formación. Sin embargo, es muy posible que subsistan otros, por lo que resulta indispensable que ellos sean advertidos por los profesores, cuando detecten que determinadas capacidades requeridas para sus módulos no han sido logradas en los estudiantes, por no estar previstas en módulos previos. También ha de tenerse en consideración la opinión de las Directoras de Escuela respecto de las dificultades que se van planteando en la administración de los planes de formación. En definitiva, vuelve a resultar vital la evaluación regular, periódica y participativa de la implementación curricular.

\section{Condicionantes del desarrollo de competencias}

Número de estudiantes por sección

Durante la discusión del rediseño curricular, tanto a nivel institucional como en la Carrera, se tuvo presente que uno de los elementos favorecedores de la transformación curricular sería el trabajo con secciones de estudiantes de acotado número (concretamente, entre 30 y 40 estudiantes). Sin embargo, este "rango ideal" solo aparece indicado en presentaciones de socialización del rediseño, pero no consta en ningún documento oficial que haya sido debidamente sancionado por la Universidad.

Con todo, parece difícil defender a ultranza la determinación de un número ideal de estudiantes por sección, y menos generalizar dicho número para hacerlo exigible a todas las carreras e incluso a todos los módulos de una misma carrera. En respeto de la lógica del modelo, el criterio rector debe ser nuevamente el de las competencias comprometidas por los distintos módulos. Mientras en algunos casos será estrictamente necesario el trabajo con grupos pequeños (ej. talleres procedimentales, módulos de integración), en otros la concentración de más estudiantes podrá resultar compatible con el logro de las competencias (ej. aquellas de fuerte componente cognitivo).

Ahora bien, si incluso se asume la distinción anterior el número de estudiantes representa una traba para el logro efectivo de las competencias de un determinado módulo, este factor desencadenará consecuencias negativas en la calidad de la docencia y en el buen desarrollo del proceso de aprendizaje (ej. dificultades de realización de determinadas estrategias metodológicas, o imposibilidad de dirección y control real del trabajo autónomo de los estudiantes).

En los hechos, las Escuelas han enfrentado dificultades para cumplir con este requerimiento operacional del rediseño. Por cierto, en algunos casos se supera el rango considerado ideal. Diversos factores interfieren en esta materia, en donde son especialmente determinantes las vacantes ofrecidas para primer año (fijadas institucionalmente), los atochamientos de estudiantes en módulos de niveles superiores (ej. en módulos de integración), y las restricciones presupuestarias. 
Exigencia de asistencia a clases

Actualmente en la Carrera se plantean las siguientes exigencias de asistencia:

Módulos sin régimen especial

Clases teóricas:

- Primer año (formación básica y disciplinaria): 80\% de asistencia.

- Segundo año (formación básica y disciplinaria): 60\% de asistencia.

- Demás niveles (formación básica y disciplinaria): asistencia libre.

Clases prácticas:

- Evaluadas: $100 \%$ de asistencia.

- No evaluadas: $90 \%$ de asistencia.

En ambos casos, el profesor debe explicitar con antelación y publicidad suficientes las sesiones en que se realizarán actividades prácticas, así como las consecuencias de la inasistencia del estudiante a actividades prácticas no evaluadas. En el caso de las actividades prácticas evaluadas, no serán recuperables y la inasistencia supondrá la calificación mínima en ellas.

Módulos con régimen especial

- Talleres: $90 \%$ de asistencia.

- Módulos de integración: 100\% de asistencia.

- Clínica Jurídica: porcentaje exigido en syllabus y Reglamento específico.

Los criterios referidos son adecuados. Efectivamente los estudiantes iniciales requieren, en general, de un proceso formativo más guiado, y en esa perspectiva resulta coherente la exigencia de asistencia obligatoria a un elevado porcentaje de actividades presenciales. La misma lógica subyace en la propia distribución entre actividades presenciales y no presenciales en módulos de distintos niveles del itinerario curricular.

También es consistente con el modelo que la definición de porcentajes de asistencia obligatoria en módulos de niveles superiores a primero se haga en razón de los requerimientos propios de cada uno de ellos.

Exigencia de nota 4.0 en productos finales esperados de los módulos

En general, para la aprobación de los módulos se exigía obtener al menos nota 4,0 en el producto final esperado de los mismos, en tanto era requerimiento aprobatorio debidamente explicitado en los syllabus ${ }^{11}$.

Esta exigencia se relacionaba con la propia existencia y naturaleza de los productos finales. En efecto, cuando el producto final es demasiado relevante para evaluar el logro de las competencias que compromete el módulo, pueden tomarse diversas decisiones. Entre ellas, asignar mayor ponderación a esta evaluación respecto del promedio del módulo, establecer

\footnotetext{
${ }^{11}$ La escala de calificaciones que utiliza la Universidad de Talca va de 1,0 a 7,0.
} 
condiciones o requerimientos de presentación (ej. nota mínima de presentación o tener aprobados todos los subproductos), o exigir la satisfacción de un estándar aprobatorio en esta actividad.

Concretamente, la exigencia de nota 4,0 en el producto final se justificaba cuando la(s) competencia(s) más compleja(s) que se evalúa mediante el producto esperado del módulo reclama la integración de las capacidades que componen las distintas unidades de aprendizaje. Es decir, en la lógica del modelo de educación basada en competencias el objetivo de este requerimiento no es añadir dificultad a la aprobación del módulo, sino permitir la verificación de la integración al interior del mismo, cuando así se desprende de las competencias comprometidas. Por tanto, en dichos casos es preciso verificar el logro de esas competencias integradoras, no siendo bastante el logro de competencias o capacidades parciales secuenciadas.

En otras palabras, hay ocasiones en que las capacidades que integran una competencia tienen carácter no-compensatorio, lo que indica que no es posible proceder por simple promedio para determinar aritméticamente que un estudiante la ha logrado. Dentro de esta perspectiva, la exigencia pierde sentido respecto de aquellos módulos en que las competencias comprometidas no requieren el ejercicio de integración interna antes referido, va bastando en estos supuestos verificar el logro de las competencias que componen cada unidad de aprendizaje.

Atendidas las consideraciones anteriores, el Consejo de Facultad ha eliminado recientemente esta exigencia, manteniéndose sólo respecto de los módulos de integración, Seminario de Investigación y Clínica Jurídica.

Metodologías y herramientas docentes

Como se ha venido comentando, el modelo de formación basada en competencias supone innovaciones no sólo metodológicas, sino una reforma desde la propia base de los perfiles de licenciatura y modificaciones sustantivas a los planes de formación. No obstante, sigue siendo un error común circunscribir el rediseño curricular casi exclusivamente a la reformulación de las prácticas docentes y a la innovación metodológica, y asumir que sin innovación en esta materia no hay rediseño ${ }^{12}$. Las consecuencias de esta concepción imprecisa pueden ser nefastas, al punto de llegar a forzar la incorporación de actividades prácticas en todos los módulos.

Por cierto que la innovación en esta materia es muy relevante. Efectivamente, el modelo supone el diseño de nuevos ambientes y situaciones de aprendizaje coherentes con las competencias que se desea desarrollar en los estudiantes y futuros profesionales. Es decir, toda innovación docente debe ser consistente con competencias definidas para el módulo respectivo. Por tanto, podrá haber módulos que en razón de las competencias que compromete no requieren gran innovación metodológica.

\footnotetext{
${ }^{12}$ Respecto de docencia universitaria y qué elementos distinguen al buen docente, Véase ASÚN, R., RUIZ, S., RETAMAL, H., PERALTA, M., ESQUIVEL, A., VARGAS, L., y MARTÍNEZ, F. "El Desafío de Educar a Inicios del Siglo XXI: Demandas de los Estudiantes de Ciencias Sociales a la Docencia Universitaria". Estudios Pedagógicos, 2013, vol.39, núm. 1. Valdivia. P. 45-62.
} 
En cualquier caso, la redefinición de estrategias metodológicas en atención a las competencias pretendidas no se agota en la definición de nuevas actividades, sino en repensar la mejor forma de conseguir los propósitos de aprendizaje. Y en esta perspectiva es la experiencia la que se intenciona para que los estudiantes lleguen a lograr una determinada capacidad.

En tanto las estrategias metodológicas deben comprometerse en los syllabus, las actividades concretas se definen en el plan de clases que lleva cada profesor. Por ejemplo, una estrategia metodológica es la discusión socializada, que puede concretarse en actividades diversas, como debates colectivos en aula, lluvia de ideas o participación en foros virtuales.

La renovación didáctica requiere, en definitiva, pasar de la enseñanza basada en los contenidos a la enseñanza basada en competencias, de lo que es capaz de reproducir o aplicar el estudiante, a las acciones que revelan que domina una competencia determinada. Es evidente, por tanto, que el rediseño exige una mayor vinculación del profesor con el proceso educativo, cuestión que no siempre se da, por factores diversos (ej. distintos vínculos contractuales y dedicaciones horarias). Pero la renovación metodológica también supone una mayor demanda de trabajo para los estudiantes, tanto en aula como fuera de ella.

Ahora bien, los principales problemas que en esta materia ha evidenciado la implementación del rediseño en la Carrera se ubican en dos dimensiones: primero, en la propia definición de las estrategias metodológicas adecuadas para la consecución de las competencias de cada módulo y, segundo, en la verificación de su efectivo desarrollo una vez definidas.

Respecto de lo primero, puede percibirse que no siempre se otorga la relevancia debida a la determinación de "estrategias y procedimientos metodológicos", exigida por el formato institucional de syllabus. No es difícil encontrar, por ejemplo, syllabus que reproducen las mismas estrategias metodológicas respecto de todas sus unidades de aprendizaje, pese a que ellas comprometen capacidades sustancialmente diferentes.

Por su parte, también se plantean problemas a la hora de intentar verificar el efectivo desarrollo de las estrategias metodológicas definidas en los syllabus. En efecto, los instrumentos de verificación existentes resultan insuficientes: a) declaración docente que consta en el syllabus, b) libro registro de clases, con indicación de si sesión será teórica o práctica, y c) evaluación docente institucional y encuesta que aplica la Escuela.

\section{Instrumentos de verificación del logro de competencias}

\section{Módulos de integración}

Los Planes de Formación 44, 11 y 15 contemplan tres módulos de integración dentro del itinerario curricular. Dichas instancias se consideran tras el cuarto, sexto y octavo semestre, con el propósito de servir de hilo conductor de la evolución multidisciplinaria del estudiante, va verificando y favoreciendo la adquisición sistemática y gradual de los componentes de competencias. 
En efecto, los módulos de integración son necesarios para la comprobación de las competencias en hitos intermedios de la hipótesis evolutiva del perfil. Además, debe brindarse a los estudiantes la oportunidad de realizar ejercicios de integración antes del egreso; no resultaría coherente exigírselos para licenciarse si no han podido practicarlos y aprender de dichas experiencias.

Cabe recordar que en la génesis del Plan de Formación 44 se definieron, además del perfil del licenciado, los perfiles por nivel, concebidos como instrumentos que permitiesen ir evaluando el logro progresivo de las competencias previstas en la hipótesis evolutiva de los estudiantes. Estos perfiles, que fueron vitales en el proceso de modularización, pueden ser una buena guía en la construcción de los módulos de integración y en la definición de las estrategias metodológicas más apropiadas a sus propósitos de aprendizaje.

Efectivamente, enfocar los módulos de integración al logro de estos perfiles intermedios puede disminuir el riesgo de distinguir las instancias integradoras en razón de las disciplinas jurídicas estudiadas en el respectivo nivel, y no en razón de las competencias más complejas que el solo saber disciplinar- que hasta ese momento deberían haber adquirido los estudiantes.

Ahora bien, dado que estos perfiles no se han redefinido tras las modificaciones sucesivas al Plan de Formación 44, ni tras la aprobación del Plan 11 y sus modificaciones, en la actualidad no se corresponden completamente con las competencias comprometidas por cada nivel o año de Carrera. Por tanto, se sugiere redefinir los perfiles por nivel, en el sentido de adecuarlos a las modificaciones que han sufrido planes de formación.

En cuanto a la operatividad de estos módulos, ha sido una buena decisión ubicarlos fuera del período lectivo, en modalidad concentrada, en cuanto permite dedicación exclusiva de los estudiantes y facilita su administración. También, parece una buena idea la de recurrir al apoyo de abogados con experiencia en el ejercicio de la profesión; pueden favorecer una visión más realista del fenómeno jurídico y la identificación de interesantes problemas jurídicos prácticos. Ahora, conviene que la coordinación de estos módulos se mantenga en profesores de planta o con un alto grado de vinculación con la Escuela, a fin de resguardar el cumplimiento de sus propósitos, por cierto intencionados dentro del Plan de Formación.

\section{Examen de Licenciatura}

\section{La concepción original de la Actividad Final de Licenciatura (en adelante, AFL)}

La AFL constituía el segundo ciclo de los Planes de Formación 44 y 11, en sus versiones originales, va teniendo asignados 30 y 27 créditos ECTS, respectivamente, distribuidos en un 1 semestre académico, con actividad presencial y no presencial.

La AFL se justificaba a partir del propio plan de formación en base a competencias profesionales, el que supone la medición y evaluación permanente, tanto de los logros de niveles intermedios, como especialmente de los logros de salida, esto es, la verificación del perfil de licenciatura.

Hasta el décimo semestre, los planes de formación 44 y 11 contemplaban diversos módulos de las áreas de formación fundamental, básica y disciplinaria, algunos con visión particular 
y otros con visión integradora de las diversas competencias y capacidades que conforman nuestro perfil. Sin embargo, terminado el décimo semestre, se hacía necesaria una instancia o actividad final que permitiese verificar el logro, por cada estudiante, del perfil de competencias definido para el licenciado.

En esta perspectiva, la Facultad justificó la AFL ante el Consejo Académico de la Universidad en base a criterios de pertinencia, relevancia y utilidad en razón del modelo educativo adoptado:

a) Pertinencia. El itinerario curricular no contemplaba -ni contempla actualmente- un módulo de integración tras el quinto año. Sin embargo, era necesario incluir en el itinerario curricular, una vez aprobados los módulos de formación fundamental, básica y disciplinaria, una actividad final de integración, comprensiva de los saberes disciplinarios, procedimentales y actitudinales más relevantes para desempeñar exitosamente las tareas profesionales básicas exigibles a un recién licenciado. Lo contrario implicaría, para la Universidad de Talca, la imposibilidad de certificar adecuadamente ante la sociedad que nuestros licenciados en Ciencias Jurídicas han adquirido las complejas competencias predefinidas en nuestro perfil.

La AFL fue concebida, entonces, como el nexo que daba cuenta de la coherencia entre los módulos de formación y el perfil de licenciatura.

b) Relevancia. La AFL era relevante, en primer término, para la consecución de los objetivos de enseñanza-aprendizaje declarados por la Carrera, por cuanto pretendía dar cuenta de los más altos niveles de integración, síntesis y maestría logrados por nuestros estudiantes de pregrado. Asimismo, era relevante para los propios estudiantes, pues con el desarrollo de esta actividad se les otorgaba la oportunidad de apropiarse de sus logros, va adquiriendo la seguridad y confianza en sí mismos, cuestión que potencia un desempeño profesional exitoso.

c) Utilidad. Por último, la Facultad justificó la necesidad de la AFL en razón de su utilidad tanto para la superación de ciertos problemas que planteaba el Plan 992 (anterior al rediseño), como para el plan de formación rediseñado.

En relación con lo primero, reemplazaba al examen de grado (tradicional en las carreras de Derecho chilenas) y generaba consistencia entre el plan de formación ofrecido al mercado y la duración real de la carrera, pues se proyectaba como una actividad guiada y enmarcada temporalmente. En relación con lo segundo, por un lado constituía una oportunidad formativa de fortalecimiento de los conocimientos, destrezas, habilidades y actitudes esenciales para el desempeño profesional. Y por otro lado, permitía la verificación del logro del perfil, va cumpliendo también una función sumativa.

En la referida presentación al Consejo Académico, la Facultad explicitó que la AFL se planteaba como un conjunto integrado de actividades tendientes a medir y evaluar en los estudiantes:

a. Los distintos niveles del desarrollo del pensamiento (conocer, comprender, analizar, y aplicar), respecto de las áreas disciplinarias definidas como fundamentales para el desempeño de un abogado. 
b. Los principales desempeños (tareas) profesionales de un abogado, que involucran la tridimensionalidad de las competencias.

c. Las destrezas y habilidades comunicacionales (escritas, orales u otras), manifestadas en relación con los puntos anteriores.

Por su carácter sumativo, esta actividad estaba proyectada como una evaluación de salida, que concentraba los aprendizajes obtenidos por el estudiante en el período formativo, sin perjuicio de su rol formativo final, al permitir la síntesis e integración.

Esta AFL fue sustituida por un examen de Licenciatura en noviembre de 2011, sin que alcanzara a aplicarse.

\section{El examen de Licenciatura}

Diversos factores desencadenaron la sustitución de la AFL por un examen de Licenciatura, actual exigencia de graduación posterior al egreso. Relevante en este sentido fue la discusión surgida entre los profesores en torno a la definición de las concretas actividades que debían componer la AFL, las diversas dificultades proyectadas en torno a su administración en un semestre académico, y las observaciones planteadas por los estudiantes.

Las RU 915 y 916, de 1 de septiembre de 2011, suprimieron el segundo ciclo que contemplaban originalmente los Planes 44 y 11, y que comprendía la realización de la AFL. En su reemplazo, establecieron como requisito de graduación posterior al egreso la aprobación de un examen de Licenciatura, cuyos objetivos, requisitos, inscripción, disciplinas evaluadas, normas de administración, procedimiento de examinación, forma de evaluación y demás normas operacionales se contienen en el Reglamento de examen de Licenciatura, aprobado por Resolución 093 de la Facultad, de 29 de noviembre de 2011 (en adelante, REL) ${ }^{13}$.

Conforme a su reglamentación, el examen de Licenciatura, aplicable a los egresados de los Planes 44, 11 y 15, "corresponde a la actividad final integradora del Plan de Formación de la carrera de Derecho de la Universidad de Talca, y comprende un conjunto integrado de actividades tendientes a medir y evaluar en los estudiantes, los distintos niveles de desarrollo alcanzados en el razonamiento jurídico de las áreas disciplinarias fundamentales para el desempeño de un abogado, así como las destrezas y habilidades comunicacionales requeridas para un desempeño profesional competente" (art. 1.1 REL). En este sentido, se concibe como una "actividad final integradora de los aprendizajes obtenidos por el estudiante en el período formativo" que "permite apreciar la evidencia de la síntesis e integración lograda durante dicha etapa precedente", va complementando de esta forma los módulos de integración desarrollados antes del egreso (art. 1.2 REL).

\footnotetext{
${ }^{13}$ Esta Resolución fue modificada parcialmente por la Resolución 046 de la Facultad, de 19 de marzo de 2012. Téngase presente que las referencias que se plantean al EL se hacen a la normativa vigente, salvo que se explicite lo contrario.
} 
En concreto, el examen de Licenciatura contempla la realización de las siguientes actividades:

- Procedimiento de monitoreo. Pretende "cautelar la rigurosidad del proceso de preparación de los egresados", a través de la asistencia -obligatoria en a lo menos un 75\%- a sesiones de orientación dirigidas por profesores de la Facultad.

- Examen de Licenciatura propiamente tal. Se compone de dos partes:

a) Examen oral de conocimientos de las instituciones fundamentales de las disciplinas jurídicas consideradas ${ }^{14}$.

El acto de examinación es público, solemne e ininterrumpido, y tiene la siguiente estructura: 1. Exposición del examinado sobre la institución de la disciplina elegida sorteada previamente, e interrogación posterior del profesor; 2. Interrogación sobre las instituciones de Derecho Civil y Procesal que el examinado sortee en el mismo acto (art. 11 REL).

b) Examen escrito de aplicación de conocimientos.

Consiste en la solución de uno o más casos jurídicos en que el examinado evidencie la comprensión y análisis de uno o más problemas jurídicos, la apreciación de los hechos constitutivos y la correcta selección del Derecho aplicable, construido sobre la base del conocimiento disciplinario integrado y de un marco ético socialmente relevado (art. 14.2 REL).

La calificación de esta actividad final integradora se obtiene del promedio ponderado de las dos partes que componen el examen de Licenciatura propiamente tal, donde el examen oral de conocimientos representa un $60 \%$ y el examen escrito de aplicación de los mismos, un $40 \%$. El procedimiento de monitoreo, por tanto, no tiene peso asignado en la calificación de este examen, sin perjuicio de que la exigencia del mínimo de asistencia indicado a las sesiones es requisito aprobatorio.

Sobre el examen de Licenciatura cabe plantear un par de observaciones. En primer término, se define como una evidencia sintética e integradora que complementa los módulos de integración, pero difiere sustancialmente de estos. No es posible, en efecto, establecer una relación de secuencialidad, por cierto con creciente complejidad en razón de las competencias evaluadas, entre ambas actividades.

Por otra parte, hay competencias del perfil del licenciado cuyo logro no está siendo verificado por el actual examen de Licenciatura, y es complejo que ello ocurra dada su formulación. En efecto, el examen oral se limita a la evaluación de conocimientos, en una lógica tradicional similar al examen de grado, y el examen escrito circunscribe las competencias evaluadas a las que se explicitan en el art. 14.2 REL. Por tanto, en mayor o menor medida, quedan sin posibilidad de verificación de logro varias competencias del perfil definido para el licenciado.

\footnotetext{
${ }^{14}$ Las disciplinas jurídicas evaluadas -obligatorias y electivas- se establecen en el art. 3 del Reglamento vigente, y las instituciones fundamentales de las mismas se precisan en los respectivos cedularios de examinación, en los términos que ordena el art. 4 de la misma reglamentación.
} 
En definitiva, si bien el examen de Licenciatura se define como una "actividad final integradora", y plantea algunas diferencias sustanciales con el tradicional examen de grado, no permite la completa verificación del perfil del licenciado, esto es, la evaluación integrada del mismo en razón de la pluridimensionalidad de las competencias allí comprometidas.

\section{Conclusiones}

Sin perjuicio de que en el cuerpo de este trabajo se han ido planteando diversas observaciones y conclusiones, conviene presentar una síntesis de las mismas:

1) El proceso de rediseño curricular ha obedecido a una decisión estratégica de la Universidad de Talca en torno a transformar sus carreras de pregrado según un modelo de educación superior basada en competencias. Derecho debió sumarse a dicho proceso y ha asumido responsablemente el desafío, en la medida de sus reales posibilidades.

2) El rediseño curricular ha supuesto, en términos generales, la modificación de los perfiles profesionales, los planes de formación, los propósitos de aprendizaje de los módulos, la metodología de enseñanza-aprendizaje, y el sistema de medición y evaluación de estudiantes y docentes.

3) En efecto, uno de los primeros productos del rediseño en la Carrera fue el levantamiento de un nuevo perfil conceptualizado del Licenciado en Ciencias Jurídicas de la Universidad de Talca, y su desagregación en un perfil desarrollado en sus dominios, competencias y capacidades, ambos validados por el cuerpo académico de la Facultad.

4) A partir del perfil definido, el rediseño curricular en la Carrera se ha traducido a la fecha en tres planes de formación, con enfoque en un modelo de educación basado en competencias: Plan 44/2006, Plan 11/2011 y Plan 15/2015.

Las modificaciones, en general, han recaído en la determinación de prerrequisitos y creditaje asignado a ciertos módulos, en la reformulación sustancial de otros, y en la eliminación del segundo ciclo de formación contemplado originalmente, con el consecuente cambio en las exigencias de graduación. La creación del Plan 11/2011, por su parte, se debió de manera fundamental a la reformulación del PFF. En el caso del Plan 15/2015, su creación obedeció al trabajo realizado a propósito del convenio de desempeño de armonización curricular.

5) El rediseño curricular se comenzó a implementar progresivamente con el Plan de Formación 44, a partir del ingreso 2006. Por su parte, la implementación del Plan 11 comenzó el primer semestre de 2011, y la implementación del Plan 15 hizo lo propio el primer semestre de 2015.

Del proceso de implementación del rediseño curricular pueden destacarse, entre otros, los siguientes aspectos: a) Relevancia de las evaluaciones periódicas al proceso, en las que se revisa el estado de avance del rediseño y se discuten los problemas que se van detectando, en tanto se promueven las modificaciones pertinentes; y b) Acompañamiento interno e institucional decreciente a docentes. 
6) En cuanto hipótesis, el plan de formación previsto originalmente, y su concreción en un determinado itinerario curricular, se han sometido a comprobación durante el proceso de implementación del rediseño. Si bien se han detectado diversas falencias y debilidades (ej. determinación de prerrequisitos de determinados módulos), es ésta la oportunidad natural para efectuar los ajustes necesarios, como ha venido ocurriendo.

7) En relación a las condicionantes del desarrollo de competencias, cabe señalar no pueden definirse en abstracto ni plantear una generalización de las mismas respecto de todos los módulos. Por el contrario, ha de valorarse su procedencia en atención a las competencias pretendidas por cada módulo.

Lo anterior no impide que puedan adoptarse definiciones para grupos de módulos que siguen un mismo patrón de competencias o plantean similares necesidades específicas, como ocurre, por ejemplo, con la necesidad de secciones de número más reducido de estudiantes en talleres procedimentales o la exigencia de asistencia obligatoria a clases para módulos de primer año, respectivamente.

8) Por otra parte, se percibe una confusión en materia de innovación metodológica fundada en el rediseño, que es preciso clarificar. Primero, la redefinición de metodologías y herramientas docentes es sólo uno de los pilares del modelo de educación basada en competencias. Es un elemento relevante del cambio en el proceso formativo, pero no el único. Segundo, se plantean diversos problemas tanto en la definición de las estrategias metodológicas como en la verificación de su concreto desarrollo en los módulos. Al respecto, sería útil generar en la Facultad algún espacio institucionalizado para compartir prácticas docentes, como una especial modalidad de acompañamiento a profesores en esta materia

9) En la verificación del logro de las competencias comprometidas destacan particularmente dos instrumentos o instancias: los módulos de integración y el examen de Licenciatura.

Por lo que respecta al primero, los Planes de Formación 44, 11 y 15 contemplan tres módulos de integración dentro del itinerario curricular, que pretenden favorecer y permitir la comprobación sistemática y gradual de las competencias en hitos intermedios de la hipótesis evolutiva del perfil. En este sentido, los perfiles por nivel deben resultar particularmente orientadores en la construcción de estos módulos y en la definición de las estrategias metodológicas más apropiadas a sus propósitos de aprendizaje. Por ello, se sugiere redefinirlos en el sentido de adecuarlos a las modificaciones que han sufrido planes de formación.

En términos operativos, se valora positivamente la decisión ubicarlos fuera del período lectivo, en modalidad concentrada, y la posibilidad de recurrir al apoyo de abogados con experiencia en el ejercicio de la profesión, sin perjuicio de que la coordinación de estos módulos la desarrollen profesores comprometidos con el logro del perfil.

10) Por su parte, el examen de Licenciatura constituye la actual instancia final exigida para la graduación, tras haberse suprimido el segundo ciclo que contemplaban originalmente los Planes 44 y 11, y que comprendía la realización de la AFL. Este examen se encuentra 
regulado en el REL, aprobado por Resolución 093 de la Facultad, de 29 de noviembre de 2011, modificado parcialmente en marzo del año en curso.

$\mathrm{Si}$ bien tras las actividades que comprende el examen de Licenciatura hay un intento efectivo de plantearlo como una actividad integradora final, es posible advertir que no permite la completa verificación del perfil del licenciado, esto es, la evaluación integrada del mismo en razón de la pluridimensionalidad de las competencias allí comprometidas.

Finalmente, hemos de concluir que con todas estas tareas se busca posibilitar a los estudiantes mejores herramientas para sus logros académicos y profesionales.

\section{Referencias bibliográficas}

ACUÑA SAN MARTÍN, Marcela; PALOMO VÉLEZ, Rodrigo. Transformación curricular basada en competencias para la carrera de Derecho. Un cambio necesario. Talca: Editorial Universidad de Talca, 2009. 71 p.

ASÚN, R., RUIZ, S., RETAMAL, H., PERALTA, M., ESQUIVEL, A., VARGAS, L. y MARTÍNEZ, F. "El Desafío de Educar a Inicios del Siglo XXI: Demandas de los Estudiantes de Ciencias Sociales a la Docencia Universitaria”. Estudios Pedagógicos, 2013, vol.39, núm. 1. Valdivia. P. 45-62.

DONOSO, S., ARIAS, O., WEASON, M., FRITES, C. "La oferta de educación superior de pregrado en Chile desde la perspectiva territorial: inequidades y asimetrías en el mercado". Calidad en la Educación, 2012, núm. 37. P. 99-127.

DONOSO, S., SCHIEFELBEIN, E. “Análisis de los modelos explicativos de retención de estudiantes en la universidad: una visión desde la desigualdad social" Estudios pedagógicos, 2007, vol. 33, núm. 1. Valdivia. P. 7-27.

FERNANDEZ, E., STOCK, M. "Los límites de la gestión. Consideraciones sociológicas sobre la gestión universitaria en Chile y Alemania”. Revista Universum, 2007, núm. 22, vol.2. P.108-124. 